\title{
COMPUTING BORDER BASES WITHOUT USING A TERM ORDERING
}

\author{
STEFAN KASPAR
}

\begin{abstract}
Border bases, a generalization of Gröbner bases, have actively been researched during recent years due to their applicability to industrial problems. In [6] Kehrein and Kreuzer formulated the so called Border Basis Algorithm, an algorithm which allows the computation of border bases that relate to a degree compatible term ordering. In this paper we extend the original Border Basis Algorithm in such a way that also border bases that do not relate to any term ordering can be computed by it.
\end{abstract}

\section{INTRODUCTION}

Let $P=K\left[x_{1}, \ldots, x_{n}\right]$ be a polynomial ring over a field $K$ and $I \subset P$ be a zero-dimensional ideal. Let $\mathcal{O}=\left\{t_{1}, \ldots, t_{m}\right\}$ be a set of terms that is closed under taking divisors. Such a set is called order ideal and its border $\left\{b_{1}, \ldots, b_{s}\right\}=$ $\left(x_{1} \mathcal{O} \cup \ldots \cup x_{n} \mathcal{O}\right) \backslash \mathcal{O}$ is denoted by $\partial \mathcal{O}$. A set $\mathcal{G}=\left\{g_{1}, \ldots, g_{s}\right\} \subset P$ of polynomials is then called an $\mathcal{O}$-border basis of $I$ if $g_{j}=b_{j}-\sum_{i=1}^{m} c_{j i} t_{i}$ where $c_{j i} \in K, \mathcal{G}$ generates $I$, and $\mathcal{O}$ is a $K$-vector space basis of $P / I$. Border bases have actively been researched during recent years (see for instance [4] [5], 6], 7], and [9]) which is due to several reasons. One aspect is that border bases are a generalization of Gröbner bases. If we denote by $\mathcal{O}_{\sigma}\{I\}$ the complement of the set of leading terms of $I$ with respect to any term ordering $\sigma$ then there exists an (unique) $\mathcal{O}_{\sigma}\{I\}$ border basis of $I$ and this border basis also contains the reduced $\sigma$-Gröbner basis of $I$. In addition border bases not only generalize Gröbner bases but are also more suitable for computations that deal with empirical polynomials, i.e. polynomials that were constructed from measured data (cf. [11]). This advantage over Gröbner basis has even lead to industrial applications (cf. 4]).

The natural question is how border bases can actually be computed. In 6 Kehrein and Kreuzer show how it is possible to compute any $\mathcal{O}$-border basis of an ideal if the order ideal $\mathcal{O}$ is already known. The downside of this approach is that it involves the computation of a Gröbner basis of the input ideal and that $\mathcal{O}$ has to be be specified explicitly. In [9] Mourrain proposed a generic framework for computing quotient bases and generators of an ideal. This framework has been refined by Kehrein and Kreuzer in [6]. There, the so called Border Basis Algorithm is formulated which allows the explicit computation of $\mathcal{O}_{\sigma}\{I\}$-border bases where $\sigma$ is a degree compatible term ordering (cf. [6, Proposition 18]). This algorithm is the basis for the work in this paper. We deduce an extension of the Border Basis Algorithm which allows us to compute more general border bases that do not rely on a term ordering. To do so we shortly revisit the necessary theoretical

Date: May 30, 2018.

2010 Mathematics Subject Classification. 13P10. 
background about border bases at the beginning of section 2. Then we collect some results from [6] which we need to formulate our Border Basis Algorithm generalization and give an example of a border basis that cannot be computed by the Border Basis Algorithm of Kehrein and Kreuzer. In section 3 we first formalize the concept of marking polynomials. This concept allows us to speak about the marked term of a polynomial and replaces the usage of a degree compatible term ordering in the original Border Basis Algorithm. We then reformulate those parts of the original Border Basis Algorithm that need to be adapted to the concept of marked polynomials. Finally we propose a new Border Basis Algorithm that does not use a degree compatible term ordering for the computation of a border basis. We show that it really is an extension of the original Border Basis Algorithm and analyze its properties. In the final section 4 we put our new Border Basis Algorithm into context with the approaches to compute border bases of Mourrain and Trébuchet in [10] and Braun and Pokutta in [2].

Acknowledgement. The author would like to thank Prof. Dr. Martin Kreuzer of the Department of Informatics and Mathematics, Universität Passau, for fruitful discussions and helpful suggestions with respect to this paper.

\section{Basic Definitions and Results}

In the following let $K$ be a field, $n \geq 1, P=K\left[x_{1}, \ldots, x_{n}\right]$, and $\mathbb{T}^{n}=$ $\left\{x_{1}^{\alpha_{1}} \cdots x_{n}^{\alpha_{n}} \mid \alpha_{i} \geq 0,1 \leq i \leq n\right\}$. Let us recall the definition of a border basis and some basic results of border basis theory as stated in [8, Section 6.4].

Definition 2.1. A set $\mathcal{O} \subseteq \mathbb{T}^{n}$ is called order ideal if $t^{\prime} \in \mathcal{O}$ and $t \mid t^{\prime}$ imply $t \in \mathcal{O}$ where $t, t^{\prime} \in \mathbb{T}^{n}$. The set $\partial \mathcal{O}=\left(x_{1} \mathcal{O} \cup \ldots \cup x_{n} \mathcal{O}\right) \backslash \mathcal{O}$ is called the border of $\mathcal{O}$. We let $\partial \emptyset=\{1\}$.

Please note that in the following we always consider finite order ideals $\mathcal{O}$ only.

Definition 2.2. Let $\mathcal{O}=\left\{t_{1}, \ldots, t_{\mu}\right\} \subset \mathbb{T}^{n}$ be an order ideal, $\partial \mathcal{O}=\left\{b_{1}, \ldots, b_{\nu}\right\}$, $G=\left\{g_{1}, \ldots, g_{m}\right\} \subset P$, and $I \subseteq P$ be an ideal.

a) The set $G$ is called $\mathcal{O}$-border prebasis if

$$
g_{j}=b_{j}-\sum_{i=1}^{\mu} \alpha_{i j} t_{i}
$$

where $\alpha_{i j} \in K$ for $1 \leq i \leq \mu$ and $1 \leq j \leq \nu$.

b) An $\mathcal{O}$-border prebasis $G$ is called $\mathcal{O}$-border basis of $I$ if $G \subset I$ and one of the following equivalent conditions is satisfied:

(i) $\overline{\mathcal{O}}=\left\{\overline{t_{1}}, \ldots, \overline{t_{\mu}}\right\}$ is a $K$-vector space basis of $P / I$.

(ii) $I \cap\langle\mathcal{O}\rangle_{K}=\{0\}$.

(iii) $P=I \oplus\langle\mathcal{O}\rangle_{K}$.

In this case $I$ is necessarily a zero-dimensional ideal.

Proposition 2.3. Let $\mathcal{O}=\left\{t_{1}, \ldots, t_{\mu}\right\} \subset \mathbb{T}^{n}$ be an order ideal, $I \subseteq P$ be a zero-dimensional ideal and assume that $\overline{\mathcal{O}}$ is a $K$-vector space basis of $P / I$.

a) There exists a unique $\mathcal{O}$-border basis of $I$.

b) If $G$ is an $\mathcal{O}$-border prebasis and $G \subset I$ then $G$ is the $\mathcal{O}$-border basis of $I$.

c) In the setting of b), I is generated by $G$. 
The following definition and proposition are taken from [6]. They will be used to state and derive the results given in in section 3 ,

Definition 2.4. Let $F \subset P$ and $V$ be a $K$-vector subspace of $P$.

a) $F^{+}=F \cup x_{1} F \cup \cdots \cup x_{n} F$.

b) $V^{+}=V+x_{1} V+\cdots+x_{n} V$.

Proposition 2.5. Let $\mathcal{U}$ be a $K$-vector subspace of $P$. Let $\mathcal{V}$ be a $K$-vector subspace of a zero-dimensional ideal $I \subseteq P$ such that $\mathcal{V}^{+} \cap \mathcal{U}=\mathcal{V}$ and $\langle\mathcal{V}\rangle=I$. Let $\mathcal{O}=\left\{t_{1}, \ldots, t_{\mu}\right\} \subset \mathbb{T}^{n}$ be an order ideal which satisfies

$$
\mathcal{U}=\mathcal{V} \oplus\langle\mathcal{O}\rangle_{K}
$$

If $\partial \mathcal{O} \subseteq \mathcal{U}$ then there exists an $\mathcal{O}$-border basis of $I$.

The following example shows in which way the standard Border Basis Algorithm as given in [6] is limited and points out the cause of the limitation. The following sections will then address this problem.

Example 2.6. Let $K=\mathbb{Q}$ and $P=\mathbb{Q}[x, y]$. Let $\mathcal{O}=\left\{1, y, y^{2}, x, x^{2}\right\}$. The set $\left\{g_{1}, g_{2}, g_{3}, g_{4}, g_{5}\right\}=\left\{x y+x^{2}-\frac{1}{2} y^{2}-x-\frac{1}{2} y, x^{3}-x, x^{2} y-\frac{1}{2} y^{2}-\frac{1}{2} y, x y^{2}+x^{2}-\right.$ $\left.\frac{1}{2} y^{2}-x-\frac{1}{2} y, y^{3}-y\right\}$ is the $\mathcal{O}$-border basis of the vanishing ideal $I$ of the five points $(-1,1),(1,1),(0,0),(1,0),(0,-1) \in \mathbb{A}^{2}(\mathbb{Q})$. But it cannot be computed by the (Improved) Border Basis Algorithm as stated in [6 because $\operatorname{LT}_{\sigma}\left(g_{1}\right)=x^{2}$ or $\operatorname{LT}_{\sigma}\left(g_{1}\right)=y^{2}$ for any term ordering $\sigma$ on $\mathbb{T}^{2}$ which implies $\operatorname{LT}_{\sigma}(I) \cap \mathcal{O} \neq \emptyset$.

\section{A Border Basis Algorithm with Term Marking Strategy}

As we have seen in example 2.6, using a (degree compatible) term ordering on $\mathbb{T}^{n}$ may prevent certain terms in the support of a polynomial from becoming a border term. In the following we investigate the idea of exchanging the term ordering for a weaker marking on the polynomials while keeping the restriction that a term $t \in \operatorname{Supp}(f)$ of a polynomial $f$ may only be marked if $\operatorname{deg}(t)=\operatorname{deg}(f)$. This idea will then be incorporated into the Improved Border Basis Algorithm [6, Proposition 21]. As a consequence we will have to reformulate those steps of the Improved Border Basis Algorithm that rely on the usage of a (degree compatible) term ordering. Let us first specify which kind of polynomial marking we want to consider.

Definition 3.1. Let $\mathcal{F} \subseteq P \backslash\{0\}$.

a) We say that the polynomials of $\mathcal{F}$ are marked if there exists a mapping $\operatorname{MT}_{\mathcal{F}}: \mathcal{F} \rightarrow \bigcup_{f \in \mathcal{F}} \operatorname{Supp}(f)$ which satisfies $\operatorname{MT}_{\mathcal{F}}(f) \in \operatorname{Supp}(f)$ and $\operatorname{deg}\left(\operatorname{MT}_{\mathcal{F}}(f)\right)=\operatorname{deg}(f)$ for all $f \in \mathcal{F}$. We call $\operatorname{MT}_{\mathcal{F}}(f)$ the marked term of $f \in \mathcal{F}$ and $\mathrm{MT}_{\mathcal{F}}$ a marking of $\mathcal{F}$.

b) If $\mathrm{MT}_{\mathcal{F}}$ is a marking of $\mathcal{F}$ then we say that $t \in \bigcup_{f \in \mathcal{F}} \operatorname{Supp}(f)$ is marked if $t=\operatorname{MT}_{\mathcal{F}}(f)$ for an $f \in \mathcal{F}$.

Example 3.2. Let $\sigma$ be a degree compatible term ordering on $\mathbb{T}^{n}$ and let $\mathrm{LT}_{\sigma}(f)$ denote the leading term of a polynomial $f \in P$ with respect to $\sigma$. Then $\mathrm{LT}_{\sigma}$ is a marking for any $\mathcal{F} \subseteq P \backslash\{0\}$. 
Example 3.3. Let $K=\mathbb{Q}, P=\mathbb{Q}[x, y]$, and $\mathcal{F}=\left\{f_{1}, f_{2}\right\}=\left\{x^{2}+x y-y^{2}-x-\right.$ $\left.y, x^{3}-x\right\}$. If we want to mark the polynomials of $\mathcal{F}$ we have to mark the term $x^{3}$ in $f_{2}$. For the marked term of $f_{1}$ we have three different possibilities to choose from: we could either mark $x^{2}, y^{2}$ or $x y$. Please note that the term $x y$ could not appear as a marked term if we would choose a degree compatible term ordering on $\mathbb{T}^{2}$ to mark the polynomials of $\mathcal{F}$ as explained in example 3.2

Our next goal is to adapt the computation procedure [6, Lemma 12] to our setting of marked polynomials.

\section{Proposition 3.4. (Marked Interreduction)}

Let $\left\{f_{1}, \ldots, f_{s}\right\} \subset P \backslash\{0\}$ be a set of marked polynomials. Let $\bigcup_{i=1}^{s} \operatorname{Supp}\left(f_{i}\right)=$ $\left\{t_{1}, \ldots, t_{l}\right\}$ be enumerated in such a way that $\operatorname{deg}\left(t_{1}\right) \geq \ldots \geq \operatorname{deg}\left(t_{l}\right)$ holds. Consider the following algorithm.

(1) Write $f_{i}=a_{i 1} t_{1}+\ldots+a_{i l} t_{l}$ for $i=1, \ldots, s$ where $a_{i j} \in K$ and let $\mathcal{A}:=\left(a_{i j}\right) \in \operatorname{Mat}_{s, l}(K)$.

(2) Let $p_{1}, \ldots, p_{s} \in\{1, \ldots, l\}$ such that $\operatorname{MT}_{\mathcal{F}}\left(f_{i}\right)=t_{p_{i}}$ for $i=1, \ldots, s$.

(3) Repeat the following steps (4) - (6) for $i=1, \ldots, s$.

(4) If $a_{i p_{i}} \neq 0$ then replace $a_{i j}$ by $a_{i p_{i}}^{-1} \cdot a_{i j}$ for $j=1, \ldots, l$.

(5) For each $k \in\{1, \ldots, s\} \backslash\{i\}$ such that $a_{k p_{i}} \neq 0$ replace $a_{k j}$ by $a_{k j}-a_{k p_{i}} \cdot a_{i j}$ for $j=1, \ldots, l$.

(6) Check for $k=i+1, \ldots, s$ if $a_{k p_{k}}=0$. For any such $k$ determine the minimum column index $j$ such that $a_{k j} \neq 0$ if such an index exists and set $p_{k}:=j$.

(7) Let $\mathcal{F}^{\prime}=\emptyset$. For $i=1, \ldots, s$ check if $f_{i}:=a_{i 1} t_{1}+\ldots+a_{i l} t_{l} \neq 0$. For any such $f_{i}$ set $\mathcal{F}^{\prime}:=\mathcal{F}^{\prime} \cup\left\{f_{i}\right\}$ and $\operatorname{MT}_{\mathcal{F}^{\prime}}\left(f_{i}\right):=t_{p_{i}}$.

(8) Return $\mathcal{F}^{\prime}$ and $\mathrm{MT}_{\mathcal{F}^{\prime}}$.

This is an algorithm that computes a set of polynomials $\mathcal{F}^{\prime}=\left\{f_{1}^{\prime}, \ldots, f_{m}^{\prime}\right\}$ and a mapping $\operatorname{MT}_{\mathcal{F}^{\prime}}: \mathcal{F}^{\prime} \rightarrow \bigcup_{i=1}^{m} \operatorname{Supp}\left(f_{i}^{\prime}\right)$ such that the following properties hold.

a) $\left\langle f_{1}^{\prime}, \ldots, f_{m}^{\prime}\right\rangle_{K}=\left\langle f_{1}, \ldots f_{s}\right\rangle_{K}$.

b) $\mathrm{MT}_{\mathcal{F}^{\prime}}$ is a marking of $\mathcal{F}^{\prime}$.

c) $\operatorname{MT}_{\mathcal{F}^{\prime}}\left(f_{i}^{\prime}\right) \notin \operatorname{Supp}\left(f_{j}^{\prime}\right)$ for all $i, j \in\{1, \ldots, m\}$ and $i \neq j$. In particular, the marked terms of $f_{1}^{\prime}, \ldots, f_{m}^{\prime}$ are pairwise different.

Proof. All loops contained in the algorithm terminate obviously after finitely many iterations.

Claim a) holds because the only changes performed on a input polynomial are the addition of $K$-multiples of some other input polynomials or the scaling of the polynomial by a factor in $K$.

To prove claim b) we show that after each iteration of the main loop (4) - (6) the equation $\operatorname{deg}\left(t_{p_{j}}\right)=\operatorname{deg}\left(a_{j 1} t_{1}+\ldots+a_{j l} t_{l}\right)$ and the inequation $a_{j p_{j}} \neq 0$ hold for all $j \in\{1, \ldots, s\}$ such that $a_{j 1} t_{1}+\ldots+a_{j l} t_{l} \neq 0$. First we note that upon termination of the steps $(4)-(6)$ the $p_{i}$-th column of $\mathcal{A}$ contains exactly one non-zero element $a_{i p_{i}}$ if the $i$-th row of $\mathcal{A}$ is not a zero row. By initialization $\operatorname{deg}\left(a_{11} t_{1}+\ldots+a_{1 l} t_{l}\right)=\operatorname{deg}\left(t_{p_{1}}\right)$ and $a_{1 p_{1}} \neq 0$ hold during the first iteration of loop $(4)-(6)$. Assume a row $k$ is going to be reduced and it does not completely reduce to zero. Before the reduction the relation $\operatorname{deg}\left(a_{k 1} t_{1}+\ldots+a_{k l} t_{l}\right) \geq \operatorname{deg}\left(t_{p_{1}}\right)$ holds. If $a_{k p_{k}} \neq 0$ after the reduction then the equality $\operatorname{deg}\left(a_{k 1} t_{1}+\ldots+a_{k l} t_{l}\right)=\operatorname{deg}\left(t_{p_{k}}\right)$ follows directly. Otherwise the equality and $a_{k p_{k}} \neq 0$ are implied by the decreasing 
ordering of the terms $t_{1}, \ldots, t_{l}$ and the update of $p_{k}$. Now assume the $i$-th iteration of the loop (4) $-(6)$ is being executed for $i \geq 2$. A reduction of a row $k$ in step (5) where $k<i$ does not affect the element $a_{k p_{k}}$ because of $a_{i p_{k}}=0$. Thus the statements $\operatorname{deg}\left(a_{k 1} t_{1}+\ldots+a_{k l} t_{l}\right)=\operatorname{deg}\left(t_{p_{k}}\right)$ and $a_{k p_{k}} \neq 0$ are true. With an argument similar to the one in the case of the first iteration of the loop (4) - (6) we derive the conclusion that the equation $\operatorname{deg}\left(a_{k 1} t_{1}+\ldots+a_{k l} t_{l}\right)=\operatorname{deg}\left(t_{p_{k}}\right)$ and the inequation $a_{k p_{k}} \neq 0$ hold for $k>i$ upon termination of step (6) whenever $a_{k 1} t_{1}+\ldots+a_{k l} t_{l} \neq 0$. This yields the correctness of claim b).

To prove the correctness of claim c) consider $i \in\{1, \ldots, s\}$ such that the $i$-th row of $\mathcal{A}$ is not a zero row. From our observations above we conclude that $a_{i p_{i}}$ is the only non-zero element of the $p_{i}$-th column of $\mathcal{A}$. As $a_{i p_{i}}$ is the coefficient of the marked term of $a_{i 1} t_{1}+\ldots+a_{i l} t_{l}$ with respect to $\mathrm{MT}_{\mathcal{F}^{\prime}}$, claim c) is true.

Remark 3.5. Obviously the resulting marking $\mathrm{MT}_{\mathcal{F}^{\prime}}$ does not only depend on the initial marking $\mathrm{MT}_{\mathcal{F}}$ but also on the ordering of the terms $t_{1}, \ldots, t_{l}$. To gain more control over the resulting marking $\mathrm{MT}_{\mathcal{F}^{\prime}}$, step (5) can be replaced by a refined pivot element selection process.

Remark 3.6. If the value of a pivot index $p_{k}$ is updated during the execution of the algorithm in step (6) the situation might occur that the set $\left\{i \mid j \leq i \leq l\right.$ and $\left.\operatorname{deg}\left(t_{i}\right)=\operatorname{deg}\left(t_{j}\right)\right\}$ contains more than one element. This means that $p_{k}$ could be set to any value of this set since it would not change the correctness of the algorithm. If the algorithm is equipped with an additional book keeping functionality that creates a log entry whenever such a situation occurs, it is possible to perform backtracking to redo the computation with different pivot indices.

Example 3.7. Let $P=\mathbb{Q}[x, y, z]$ and $\mathcal{F}=\left\{f_{1}, f_{2}, f_{3}, f_{4}, f_{5}\right\}=\left\{x y^{2}+x^{3}+\right.$ $\left.z, x y^{2}-y z, x y^{2}+1, y^{5}-x y^{2}-y^{2}+y z+z, z^{3}\right\}$ a set of marked polynomials where $\operatorname{MT}_{\mathcal{F}}\left(f_{1}\right)=\operatorname{MT}_{\mathcal{F}}\left(f_{2}\right)=\operatorname{MT}_{\mathcal{F}}\left(f_{3}\right)=x y^{2}, \operatorname{MT}_{\mathcal{F}}\left(f_{4}\right)=y^{5}$, and $\operatorname{MT}_{\mathcal{F}}\left(f_{5}\right)=z^{3}$. We choose the numeration $t_{1}=y^{5}, t_{2}=z^{3}, t_{3}=x^{3}, t_{4}=x y^{2}, t_{5}=y z, t_{6}=y^{2}, t_{7}=z$, and $t_{8}=1$ and apply the Marked Interreduction.

(1) We obtain the matrix

$$
\mathcal{A}=\left(\begin{array}{cccccccc}
0 & 0 & 1 & {[1]} & 0 & 0 & 1 & 0 \\
0 & 0 & 0 & {[1]} & -1 & 0 & 0 & 0 \\
0 & 0 & 0 & {[1]} & 0 & 0 & 0 & 1 \\
{[1]} & 0 & 0 & -1 & 1 & -1 & 1 & 0 \\
0 & {[1]} & 0 & 0 & 0 & 0 & 0 & 0
\end{array}\right)
$$

where the elements in brackets mark the elements $a_{i p_{i}}$ in each row.

(2) We obtain $p_{1}=p_{2}=p_{3}=4, p_{4}=1$, and $p_{5}=2$.

(3) Since $a_{1 p_{1}}=1$ we do not change any element of the first row of $\mathcal{A}$.

(4) Since $a_{2 p_{1}}, a_{3 p_{1}}$, and $a_{4 p_{1}}$ are not equal to zero the matrix $\mathcal{A}$ becomes the matrix

$$
\mathcal{A}=\left(\begin{array}{cccccccc}
0 & 0 & 1 & {[1]} & 0 & 0 & 1 & 0 \\
0 & 0 & -1 & {[0]} & -1 & 0 & -1 & 0 \\
0 & 0 & -1 & {[0]} & 0 & 0 & -1 & 1 \\
{[1]} & 0 & 1 & 0 & 1 & -1 & 2 & 0 \\
0 & {[1]} & 0 & 0 & 0 & 0 & 0 & 0
\end{array}\right)
$$


(5) $a_{2 p_{2}}=a_{3 p_{3}}=0$ and we set $p_{2}=p_{3}=3$.

(3) We multiply the second row of $\mathcal{A}$ by -1 .

(4) Since $a_{3 p_{2}} \neq 0$ the matrix $\mathcal{A}$ becomes the matrix

$$
\mathcal{A}=\left(\begin{array}{cccccccc}
0 & 0 & 1 & {[1]} & 0 & 0 & 1 & 0 \\
0 & 0 & {[1]} & 0 & 1 & 0 & 1 & 0 \\
0 & 0 & {[0]} & 0 & 1 & 0 & 0 & 1 \\
{[1]} & 0 & 1 & 0 & 1 & -1 & 2 & 0 \\
0 & {[1]} & 0 & 0 & 0 & 0 & 0 & 0
\end{array}\right)
$$

(5) $a_{3 p_{3}}=0$ and we set $p_{3}=5$.

(3) Since $a_{3 p_{3}}=1$ we do not change any element of the third row of $\mathcal{A}$.

(4) Since $a_{2 p_{3}}$ and $a_{4 p_{3}}$ are not equal to zero the matrix $\mathcal{A}$ becomes the matrix

$$
\mathcal{A}=\left(\begin{array}{cccccccc}
0 & 0 & 1 & {[1]} & 0 & 0 & 1 & 0 \\
0 & 0 & {[1]} & 0 & 0 & 0 & 1 & -1 \\
0 & 0 & 0 & 0 & {[1]} & 0 & 0 & 1 \\
{[1]} & 0 & 1 & 0 & 0 & -1 & 2 & -1 \\
0 & {[1]} & 0 & 0 & 0 & 0 & 0 & 0
\end{array}\right)
$$

The matrix $\mathcal{A}$ is left unchanged during the remaining loops of the steps (3) - (5) where $i=4$ and $i=5$. Finally, in step (7) we obtain $\mathcal{F}^{\prime}=\left\{f_{1}^{\prime}, f_{2}^{\prime}, f_{3}^{\prime}, f_{4}^{\prime}, f_{5}^{\prime}\right\}=$ $\left\{x y^{2}+x^{3}+z, x^{3}+z-1, y z+1, y^{5}+x^{3}-y^{2}+2 z-1, z^{3}\right\}$ where $\operatorname{MT}_{\mathcal{F}^{\prime}}\left(f_{1}^{\prime}\right)=$ $x y^{2}, \operatorname{MT}_{\mathcal{F}^{\prime}}\left(f_{2}^{\prime}\right)=x^{3}, \operatorname{MT}_{\mathcal{F}^{\prime}}\left(f_{3}^{\prime}\right)=y z, \operatorname{MT}_{\mathcal{F}^{\prime}}\left(f_{4}^{\prime}\right)=y^{5}, \operatorname{MT}_{\mathcal{F}^{\prime}}\left(f_{5}^{\prime}\right)=z^{3}$.

Let $\mathcal{F}=\left\{f_{1}, \ldots, f_{s}\right\} \subset P \backslash\{0\}$ and assume that $I=\langle\mathcal{F}\rangle_{P}$ is zero-dimensional. We are now ready to reformulate the Improved Border Basis Algorithm 6, Proposition 21]. The result will be an algorithm that allows the user to outline a subset of $\mathbb{T}^{n}$ by choosing a marking on $\mathcal{F}$. The algorithm will then try to compute a suitable order ideal $\mathcal{O}$ in this outlined subset of $\mathbb{T}^{n}$ for which an $\mathcal{O}$-border basis of $I$ exists. This flexibility is achieved at the cost of a loss of predictability: The algorithm will not be able to produce an $\mathcal{O}$-border basis of $I$ for any given marking on $\mathcal{F}$ (cf. remark 3.15). However, if a degree compatible term ordering $\sigma$ on $\mathbb{T}^{n}$ is used to mark the polynomials of $\mathcal{F}$ and the polynomials obtained during the computation then the algorithm will always compute the $\mathcal{O}_{\sigma}\{I\}$-border basis of $I$ (cf. remark 3.14). Lastly, we note that the overall structure of the Improved Border Basis Algorithm [6, Proposition 21] will be kept unchanged in the following reformulation.

Proposition 3.8. (Border Basis Algorithm with Term Marking Strategy) Let $\mathcal{F}=\left\{f_{1}, \ldots, f_{s}\right\} \subset P \backslash\{0\}$ be a set of marked polynomials such that $\operatorname{MT}_{\mathcal{F}}\left(f_{i}\right) \notin$ $\operatorname{Supp}\left(f_{j}\right)$ where $1 \leq i, j \leq s$ and $i \neq j$. Assume that $I=\langle\mathcal{F}\rangle_{P}$ is zero-dimensional. The following algorithm stops without a result in step (T7) or computes an order ideal $\mathcal{O} \subset \mathbb{T}^{n}$ and a set of marked polynomials $\left\{g_{1}, \ldots, g_{\nu}\right\}$ such that $\left\{g_{1}, \ldots, g_{\nu}\right\}$ is the $\mathcal{O}$-border basis of $I$ and if $t \in \operatorname{Supp}(g)$ satisfies $t \in \partial \mathcal{O}$ for a $g \in\left\{g_{1}, \ldots, g_{\nu}\right\}$ then $t$ is marked in $g$.

(T1) Let $\mathcal{U}$ be the order ideal spanned by $\bigcup_{i=1}^{s} \operatorname{Supp}\left(f_{i}\right)$.

(T2) Compute a $K$-vector space basis $\mathcal{V}$ of $\langle\mathcal{F}\rangle_{K}$ with pairwise different marked terms: Apply the Marked Interreduction to $\left\{f_{1}, \ldots, f_{s}\right\}$ to obtain $\mathcal{V}=$ $\left\{f_{1}^{\prime}, \ldots, f_{m}^{\prime}\right\}$. 
(T3) Compute a $K$-vector space basis $\mathcal{V}^{\prime} \cup \mathcal{W}^{\prime}$ of $\left\langle\mathcal{V}^{+}\right\rangle_{K}$ such that the elements of $\mathcal{V}^{\prime} \cup \mathcal{W}^{\prime}$ have pairwise different marked terms and $\operatorname{MT}_{\mathcal{V}^{\prime} \cup \mathcal{W}^{\prime}}(v) \notin \operatorname{Supp}(w)$ for all $v, w \in \mathcal{V}^{\prime} \cup \mathcal{W}^{\prime}$ and $v \neq w$ :

a) Mark in all $x_{i} v \in \mathcal{V}^{+} \backslash \mathcal{V}, 1 \leq i \leq n, v \in \mathcal{V}$ the term $x_{i} t$ where $t \in \operatorname{Supp}(v)$ is the marked term of $v$.

b) Let $\mathcal{V}=\left\{v_{1}, \ldots, v_{l}\right\}$ and $\mathcal{V}^{+} \backslash \mathcal{V}=\left\{v_{1}^{\prime}, \ldots, v_{l^{\prime}}^{\prime}\right\}$ and apply the Marked Interreduction to $\left\{v_{1}, \ldots, v_{l}, v_{1}^{\prime}, \ldots, v_{l^{\prime}}^{\prime}\right\}$ to obtain $\tilde{\mathcal{V}}=\left\{\tilde{f}_{1}, \ldots, \tilde{f}_{m}\right\}$.

c) Let $T=\left\{\operatorname{MT}_{\mathcal{V}}(v) \mid v \in \mathcal{V}\right\}$ and $\mathcal{V}^{\prime}=\left\{\tilde{f} \in \tilde{\mathcal{V}} \mid \operatorname{MT}_{\tilde{\mathcal{V}}}(\tilde{f}) \in T\right\}$. Let $\mathcal{W}^{\prime}=\tilde{\mathcal{V}} \backslash \mathcal{V}^{\prime}$

(T4) Let $\mathcal{W}=\left\{w \in \mathcal{W}^{\prime} \mid \operatorname{MT}_{\mathcal{W}^{\prime}}(w) \in \mathcal{U}\right\}$

(T5) If $\bigcup_{w \in \mathcal{W}} \operatorname{Supp}(w) \nsubseteq \mathcal{U}$ then replace $\mathcal{U}$ by the order ideal spanned by $\mathcal{U}$ and $\bigcup_{w \in \mathcal{W}} \operatorname{Supp}(w)$ and continue with (T4).

(T6) If $\mathcal{W} \neq \emptyset$ then replace $\mathcal{V}$ by $\mathcal{V}^{\prime} \cup \mathcal{W}$ and continue with (T3).

(T7) Let $\mathcal{O}=\mathcal{U} \backslash\left\{\operatorname{MT}_{\mathcal{V}}(v) \mid v \in \mathcal{V}\right\}$. If $\mathcal{O}$ is not an order ideal then stop and output "O $\mathcal{O}$ is not an order ideal in step (T7).".

(T8) If $\partial \mathcal{O} \nsubseteq \mathcal{U}$ then replace $\mathcal{U}$ by the order ideal $\mathcal{U}^{+}$and continue with (T3).

(T9) Select in $\mathcal{V}$ those $g_{1}, \ldots, g_{\nu}$ which satisfy $\operatorname{MT}_{\mathcal{V}}\left(g_{i}\right) \in \partial \mathcal{O}$ where $1 \leq i \leq \nu$. Output $\left\{g_{1}, \ldots, g_{\nu}\right\}$ and its marking as well as $\mathcal{O}$.

Proof. The correctness of (T2) and (T3) is implied by proposition 3.4. Additionally, the following equality holds in (T3): $\bigcup_{v \in \mathcal{V}}\left\{\operatorname{MT}_{\mathcal{V}}(v)\right\}=\bigcup_{v \in \mathcal{V}^{\prime}}\left\{\operatorname{MT}_{\mathcal{V}^{\prime}}(v)\right\}$. The relation " $\supseteq$ " holds by construction of $\mathcal{V}^{\prime}$ and " $\subseteq$ " because of the order in which the input polynomials $\left\{v_{1}, \ldots, v_{l}, v_{1}^{\prime}, \ldots, v_{l^{\prime}}^{\prime}\right\}$ are processed by the Marked Interreduction.

The loop (T4)-(T5) is finite since each enlargement of $\mathcal{U}$ is contained in the finite order ideal spanned by $\bigcup_{v \in \mathcal{V}^{\prime} \cup \mathcal{W}^{\prime}} \operatorname{Supp}(v)$ because of $\mathcal{W} \subseteq \mathcal{W}^{\prime}$.

At the end of loop (T4)-(T5), $\left\langle\mathcal{V}^{\prime} \cup \mathcal{W}\right\rangle_{K}=\left\langle\mathcal{V}^{\prime} \cup \mathcal{W}^{\prime}\right\rangle_{K} \cap\langle\mathcal{U}\rangle_{K}$ holds: The relation " $\subseteq$ " follows by construction of $\mathcal{W}$ and step (T5). To show " $\supseteq$ " let $v=$ $\alpha_{1} v_{1}+\ldots+\alpha_{r} v_{r}+\beta_{1} w_{1}+\ldots+\beta_{l} w_{l} \in\langle\mathcal{U}\rangle_{K}$ where $\alpha_{1}, \ldots, \alpha_{r}, \beta_{1}, \ldots, \beta_{l} \in K \backslash$ $\{0\}, v_{1}, \ldots, v_{r} \in \mathcal{V}^{\prime}$, and $w_{1}, \ldots, w_{l} \in \mathcal{W}^{\prime}$. If $r+l=1$, the inclusion follows by $\operatorname{MT}_{\mathcal{V}^{\prime} \cup \mathcal{W}^{\prime}}(v) \in \mathcal{U}$. Now let $r+l>1$. If $r \geq 1$ then $v-\alpha_{1} v_{1} \in\left\langle\mathcal{V}^{\prime} \cup \mathcal{W}^{\prime}\right\rangle_{K} \cap\langle\mathcal{U}\rangle_{K}$ because of $\operatorname{Supp}\left(v_{1}\right) \subseteq \mathcal{U}$ and by the induction hypothesis $v=\left(v-\alpha_{1} v_{1}\right)+\alpha_{1} v_{1} \in$ $\left\langle\mathcal{V}^{\prime} \cup \mathcal{W}\right\rangle_{K}$. If $l \geq 1$ then $v-\beta_{1} w_{1} \in\left\langle\mathcal{V}^{\prime} \cup \mathcal{W}^{\prime}\right\rangle_{K} \cap\langle\mathcal{U}\rangle_{K}$ holds: The term $\operatorname{MT}_{\mathcal{W}^{\prime}}\left(w_{1}\right)$ neither is contained in $\operatorname{Supp}\left(v_{i}\right), 1 \leq i \leq r \operatorname{nor} \operatorname{Supp}\left(w_{i}\right), 2 \leq i \leq l$. This implies $\operatorname{MT}_{\mathcal{W}^{\prime}}\left(w_{1}\right) \in \mathcal{U}$ and therefore $w_{1} \in \mathcal{W}$ since $(\mathrm{T} 4)$ has been executed and $v-\beta_{1} w_{1} \in\left\langle\mathcal{V}^{\prime} \cup \mathcal{W}^{\prime}\right\rangle_{K} \cap\langle\mathcal{U}\rangle_{K}$ since (T5) has been executed. Again, by induction hypothesis $v=\left(v-\beta_{1} w_{1}\right)+\beta_{1} w_{1} \in\left\langle\mathcal{V}^{\prime} \cup \mathcal{W}\right\rangle_{K}$.

The loop (T3)-(T6) is finite: At the beginning of an arbitrary iteration, let $\mathcal{U}$ be contained some $\mathbb{T}_{\leq d}^{n}$. A possible enlargement of $\mathcal{U}$ in (T3) is contained in $\mathbb{T}_{\leq d}^{n}$. The subset selection criterion $\operatorname{MT}_{\mathcal{W}^{\prime}}(w) \in \mathcal{U}$ in $(\mathrm{T} 4)$ yields $\operatorname{Supp}(w) \subseteq \mathbb{T}_{\geq d}^{n}$ where $w \in \mathcal{W}^{\prime}$ since $\operatorname{deg}\left(\operatorname{MT}_{\mathcal{W}^{\prime}}(w)\right)=\operatorname{deg}(w)$ holds. Thus all enlargements of $\mathcal{U}$ take place in the finite set $\mathbb{T}_{\leq d}^{n}$.

Upon termination of loop (T3)-(T6), the equality $\langle\mathcal{V}\rangle_{K}=\langle\mathcal{V}\rangle_{K}^{+} \cap\langle\mathcal{U}\rangle_{K}$ holds: (T3) yields $\left\langle\mathcal{V}^{\prime} \cup \mathcal{W}^{\prime}\right\rangle_{K}=\langle\mathcal{V}\rangle_{K}^{+}$. Since the loop (T4)-(T5) has been executed, $\left\langle\mathcal{V}^{\prime} \cup \mathcal{W}\right\rangle_{K}=\left\langle\mathcal{V}^{\prime} \cup \mathcal{W}^{\prime}\right\rangle_{K} \cap\langle\mathcal{U}\rangle_{K}$. After exiting the loop (T3)-(T6) in (T6), $\mathcal{W}=\emptyset$ and therefore $\left\langle\mathcal{V}^{\prime}\right\rangle_{K}=\langle\mathcal{V}\rangle_{K}^{+} \cap\langle\mathcal{U}\rangle_{K}$. It remains to show $\langle\mathcal{V}\rangle_{K}=\left\langle\mathcal{V}^{\prime}\right\rangle_{K}$. This 
is implied by $\bigcup_{v \in \mathcal{V}}\left\{\operatorname{MT}_{\mathcal{V}}(v)\right\}=\bigcup_{v \in \mathcal{V}^{\prime}}\left\{\operatorname{MT}_{\mathcal{V}^{\prime}}(v)\right\}$ and $\mathcal{W}=\emptyset$ during the last iteration of (T3)-(T6).

The loop (T3)-(T8) is finite: Consider the case that the algorithm does not terminate in any iteration in (T7). Let $i \in\{1, \ldots, n\} . I$ is zero-dimensional thus $I \cap K\left[x_{i}\right] \neq \emptyset$, i.e. there exists a $p=x_{i}^{d}+\ldots+a_{1} x_{i}+a_{0} \in I$ where $d \in \mathbb{N}$ and $a_{0}, \ldots, a_{d-1} \in K$. Let $p=h_{1} f_{1}+\ldots+h_{s} f_{s}$ where $h_{j} \in P$ where $1 \leq j \leq s$. Since each execution of (T8) strictly enlarges $\mathcal{U}$, the relation $h_{j} f_{j} \in\langle\mathcal{U}\rangle_{K}$ must hold for all $j \in\{1, \ldots, s\}$ after finitely many iterations which yields $p \in\langle\mathcal{V}\rangle_{K}$. Now let $p=x_{i}^{d}+\ldots+a_{1} x_{i}+a_{0}=b_{1} v_{1}+\ldots+b_{r} v_{r}$ where $b_{1}, \ldots, b_{r} \in K \backslash\{0\}$ and $v_{1}, \ldots, v_{r} \in \mathcal{V}$. Then $x_{i}^{d}=\operatorname{MT}_{\mathcal{V}}\left(v_{j}\right)$ for a $j \in\{1, \ldots, r\}$ : Assume for a contradiction $x_{i}^{d} \neq \operatorname{MT}_{\mathcal{V}}\left(v_{j}\right)$ for all $j \in\{1, \ldots, r\}$. By construction, $\operatorname{MT}_{\mathcal{V}}\left(v_{j}\right) \notin$ $\operatorname{Supp}\left(v_{k}\right)$ where $j, k \in\{1, \ldots, r\}$ and $j \neq k$. This yields $\operatorname{MT}_{\mathcal{V}}\left(v_{j}\right) \in \operatorname{Supp}(p) \backslash\left\{x^{d}\right\}$. Thus $\operatorname{deg}\left(v_{j}\right)=\operatorname{deg}\left(\operatorname{MT}_{\mathcal{V}}\left(v_{j}\right)\right)<d$ where $1 \leq j \leq r$ in contradiction to $\operatorname{deg}(p)=$ $\operatorname{deg}\left(b_{1} v_{1}+\ldots+b_{r} v_{r}\right)=d$. Since no marked term will vanish in a future iteration of loop (T3)-(T8) we conclude that for all $i \in\{1, \ldots, n\}$ there exist powers $d_{i} \in \mathbb{N}$ s.t. $x_{1}^{d_{1}}, \ldots, x_{n}^{d_{n}}$ appear as marked terms of polynomials of $\mathcal{V}$ in some iteration. The order ideals $\mathcal{O}$ constructed in (T7) contain no multiples of marked terms. Thus the growth of the order ideals $\mathcal{O}$ is bounded since $\mathbb{T}^{n} \backslash\left\{t \cdot x_{i}^{d_{i}} \mid t \in \mathbb{T}^{n}, 1 \leq i \leq n\right\}$ is finite. Therefore after finitely many iterations the relation $\partial \mathcal{O} \subseteq \mathcal{U}$ in (T8) must hold and the loop terminates.

Finally, assume that (T9) is being executed: By proposition 2.5 where $\tilde{I}=\langle\mathcal{V}\rangle_{K}$ the $\mathcal{O}$-border basis of $I$ exists. By construction, $\operatorname{Supp}(v) \backslash\left\{\mathrm{MT}_{\mathcal{V}}(v)\right\} \subseteq \mathcal{O}$ for all $v \in \mathcal{V}$. Thus the selected $g_{1}, \ldots, g_{\nu}$ form a $\mathcal{O}$-border prebasis of $I$. Proposition 2.3 now implies that $\left\{g_{1}, \ldots, g_{\nu}\right\}$ is the $\mathcal{O}$-border basis of $I$.

Remark 3.9. Let $\mathcal{F}^{\prime}=\left\{f_{1}^{\prime}, \ldots, f_{s^{\prime}}^{\prime}\right\} \subset P \backslash\{0\}$ be a set of marked polynomials such that $\left\langle\mathcal{F}^{\prime}\right\rangle_{P}$ is zero-dimensional. Using the Marked Interreduction, $\mathcal{F}^{\prime}$ can easily be transformed into a set $\mathcal{F}=\left\{f_{1}, \ldots, f_{s}\right\} \subset P \backslash\{0\}$ that fulfills the precondition $\operatorname{MT}_{\mathcal{F}}\left(f_{i}\right) \notin \operatorname{Supp}\left(f_{j}\right)$ where $1 \leq i, j \leq s$ and $i \neq j$ of the Border Basis Algorithm with Term Marking Strategy.

Remark 3.10. The following examples shows that this variant of the Improved Border Basis Algorithm indeed allows the computation of border bases that cannot be computed by the standard (Improved) Border Basis Algorithm.

Example 3.11. Let $K=\mathbb{Q}$ and $P=\mathbb{Q}[x, y]$. Let $\mathcal{F}=\left\{f_{1}, f_{2}, f_{3}\right\}=\left\{x^{2}+x y-\right.$ $\left.\frac{1}{2} y^{2}-x-\frac{1}{2} y, y^{3}-y, x y^{2}-x y\right\}$. The ideal $I=\left\langle f_{1}, f_{2}, f_{3}\right\rangle$ is equal to the vanishing ideal of the five points $(-1,1),(1,1),(0,0),(1,0),(0,-1) \in \mathbb{A}^{2}(\mathbb{Q})$ of example 2.6. We apply algorithm 3.8 to $\mathcal{F}$.

(T1) The computing universe $\mathcal{U}$ is equal to $\left\{1, x, y, x^{2}, x y, y^{2}, x y^{2}, y^{3}\right\}$.

(T2) We may only choose the marked term of $f_{1}$ freely because the degree restriction forces us to mark $y^{3}$ in $f_{2}$ and $x y^{2}$ in $f_{3}$. Since $\operatorname{deg}\left(f_{1}\right)=2$, one of the terms $x^{2}, x y$ or $y^{2}$ can be marked in $f_{1}$. We choose $x y$.

We apply the Marked Interreduction to $\mathcal{F}$ and choose the numeration $t_{1}=y^{3}, t_{2}=x y^{2}, t_{3}=x y, t_{4}=x^{2}, t_{5}=y^{2}, t_{6}=x$, and $t_{7}=y$. We obtain $f_{1}^{\prime}=x y+x^{2}-\frac{1}{2} y^{2}-x-\frac{1}{2} y$ with marked term $x y, f_{2}^{\prime}=y^{3}-y$ with marked $y^{3}$, and $f_{3}^{\prime}=x y^{2}+x^{2}-\frac{1}{2} y^{2}-x-\frac{1}{2} y$ with marked term $x y^{2}$. We let $\mathcal{V}=\left\{f_{1}^{\prime}, f_{2}^{\prime}, f_{3}^{\prime}\right\}$.

(T3) a) We mark $x^{2} y$ in $x v_{1}, x y^{2}$ in $y v_{1}, x y^{3}$ in $x v_{2}, y^{4}$ in $y v_{2}, x^{2} y^{2}$ in $x v_{3}$, and $x y^{3}$ in $y v_{3}$. 
b) We let $\mathcal{V}=\left\{v_{1}, v_{2}, v_{3}\right\}=\left\{f_{1}, f_{2}, f_{3}\right\}$, and $\mathcal{V}^{+} \backslash \mathcal{V}=\left\{v_{1}^{\prime}, v_{2}^{\prime}, v_{3}^{\prime}, v_{4}^{\prime}, v_{5}^{\prime}\right.$, $\left.v_{6}^{\prime}\right\}=\left\{x v_{1}, y v_{1}, x v_{2}, y v_{2}, x v_{3}, y v_{3}\right\}$. We apply the Marked Interreduction to $\left\{v_{1}, v_{2}, v_{3}, v_{1}^{\prime}, v_{2}^{\prime}, v_{3}^{\prime}, v_{4}^{\prime}, v_{5}^{\prime}, v_{6}^{\prime}\right\}$ and choose the numeration $t_{1}=x y^{3}, t_{2}=$ $y^{4}, t_{3}=x^{2} y^{2}, t_{4}=y^{3}, t_{5}=x y^{2}, t_{6}=x^{2} y, t_{7}=x y, t_{8}=x^{3}, t_{9}=x^{2}, t_{10}=$ $y^{2}, t_{11}=x$, and $t_{12}=y$ and obtain $f_{1}^{\prime}=x y+x^{2}-\frac{1}{2} y^{2}-x-\frac{1}{2} y$ with marked term $x y, f_{2}^{\prime}=y^{3}-y$ with marked term $y^{3}, f_{3}^{\prime}=x y^{2}+x^{2}-\frac{1}{2} y^{2}-x-\frac{1}{2} y$ with marked term $x y^{2}, f_{4}^{\prime}=x^{2} y-\frac{1}{2} y^{2}-\frac{1}{2} y$ with marked term $x^{2} y, f_{5}^{\prime}=x^{3}-x$ with marked term $x^{3}, f_{6}^{\prime}=x y^{3}+x^{2}-\frac{1}{2} y^{2}-x-\frac{1}{2} y$ with marked term $x y^{3}, f_{7}^{\prime}=y^{4}-y^{2}$ with marked term $y^{4}$, and $f_{8}^{\prime}=x^{2} y^{2}-\frac{1}{2} y^{2}-\frac{1}{2} y$ with marked term $x^{2} y^{2}$.

c) We let $\mathcal{V}^{\prime}=\left\{f_{1}^{\prime}, f_{2}^{\prime}, f_{3}^{\prime}\right\}$ and $\mathcal{W}^{\prime}=\left\{f_{4}^{\prime}, f_{5}^{\prime}, f_{6}^{\prime}, f_{7}^{\prime}, f_{8}^{\prime}\right\}$.

(T4) $\mathcal{W}=\emptyset$ because of $x^{2} y, x^{3}, x y^{3}, y^{4}, x^{2} y^{2} \notin \mathcal{U}$.

(T5) We continue with (T6).

(T6) We continue with (T7).

(T7) $\mathcal{O}=\left\{1, x, y, x^{2}, x y, y^{2}, x y^{2}, y^{3}\right\} \backslash\left\{x y, y^{3}, x y^{2}\right\}=\left\{1, x, y, x^{2}, y^{2}\right\}$.

(T8) $\partial \mathcal{O}=\left\{x^{3}, x^{2} y, x y, x y^{2}, y^{3}\right\} \nsubseteq \mathcal{U}$. We enlarge $\mathcal{U}$ to $\mathcal{U}^{+}=\left\{1, x, y, x^{2}, x y\right.$, $\left.y^{2}, x^{3}, x^{2} y, x y^{2}, y^{3}, x^{2} y^{2}, x y^{3}, y^{4}\right\}$ and repeat (T3)-(T8).

(T3) We obtain the same results as in (T3) above.

(T4) $\mathcal{W}=\left\{x^{2} y-\frac{1}{2} y^{2}-\frac{1}{2} y, x^{3}-x, x y^{3}+x^{2}-\frac{1}{2} y^{2}-x-\frac{1}{2} y, y^{4}-y^{2}, x^{2} y^{2}-\frac{1}{2} y^{2}-\right.$ $\left.\frac{1}{2} y\right\}=\mathcal{W}^{\prime}$

(T5) It is not necessary to enlarge $\mathcal{U}$ thus we continue with (T6).

(T6) Since $\mathcal{W} \neq \emptyset$ we replace $\mathcal{V}$ by $\left\{x y+x^{2}-\frac{1}{2} y^{2}-x-\frac{1}{2} y, y^{3}-y, x y^{2}+x^{2}-\frac{1}{2} y^{2}-\right.$ $\left.x-\frac{1}{2} y, x^{2} y-\frac{1}{2} y^{2}-\frac{1}{2} y, x^{3}-x, x y^{3}+x^{2}-\frac{1}{2} y^{2}-x-\frac{1}{2} y, y^{4}-y^{2}, x^{2} y^{2}-\frac{1}{2} y^{2}-\frac{1}{2} y\right\}$ and repeat (T3)-(T6). This will lead to $\mathcal{W}=\emptyset$ in (T6) and we leave the loop (T3)-(T6).

(T7) $\mathcal{O}=\left\{1, x, y, x^{2}, x y, y^{2}, x^{3}, x^{2} y, x y^{2}, y^{3}, x^{2} y^{2}, x y^{3}, y^{4}\right\} \backslash\left\{x y, y^{3}, x y^{2}, x^{2} y\right.$, $\left.x y^{3}, y^{4}, x^{2} y^{2}, x^{3}\right\}=\left\{1, x, y, x^{2}, y^{2}\right\}$.

(T8) $\partial \mathcal{O}=\left\{x^{3}, x^{2} y, x y, x y^{2}, y^{3}\right\} \subseteq \mathcal{U}$ and we leave the loop (T3)-(T8).

(T9) We select $g_{1}=x^{3}-x, g_{2}=x^{2} y-\frac{1}{2} y^{2}-\frac{1}{2} y, g_{3}=x y+x^{2}-\frac{1}{2} y^{2}-x-\frac{1}{2} y, g_{4}=$ $x y^{2}+x^{2}-\frac{1}{2} y^{2}-x-\frac{1}{2} y$, and $g_{5}=y^{3}-y$ with marked terms $x^{3}, x^{2} y, x y$, $x y^{2}$, and $y^{3}$ from $\mathcal{V}$ and output $g_{1}, \ldots, g_{5}$ as well as $\mathcal{O}=\left\{1, x, y, x^{2}, y^{2}\right\}$.

Finally, we note that we obtained the $\mathcal{O}$-border basis of the ideal $I$ of example 2.6 which cannot be computed by the (Improved) Border Basis Algorithm.

Example 3.12. Let $K=\mathbb{Q}, P=\mathbb{Q}[x, y, z]$, and $\mathcal{F}=\left\{f_{1}, f_{2}, f_{3}\right\}=\left\{x^{3}+x-1\right.$, $\left.y^{2}+y z+z^{2}+x z+x^{2}, z^{3}+x^{2} z+x y z-y\right\}$ be a set of marked polynomials where $\operatorname{MT}_{\mathcal{F}}\left(f_{1}\right)=x^{3}, \operatorname{MT}_{\mathcal{F}}\left(f_{2}\right)=x z$, and $\operatorname{MT}_{\mathcal{F}}\left(f_{3}\right)=x^{2} z$. The ideal $I=\left\langle f_{1}, f_{2}, f_{3}\right\rangle$ is zero-dimensional and $\operatorname{dim}_{\mathbb{Q}}(P / I)=18$. We apply algorithm 3.8 to $\mathcal{F}$ by using the DegLex term ordering on $\mathbb{T}^{3}$ to enumerate the terms of the sets $\left\{t_{1}, \ldots, t_{l}\right\}$ each time the Marked Interreduction is executed. Then the computation yields an $\mathcal{O}$-border basis of $I$ where $\mathcal{O}=\left\{1, z, y, x, z^{2}, y z, y^{2}, x y, x^{2}, z^{3}, y z^{2}, y^{2} z, y^{3}\right.$, $\left.x^{2} y, z^{4}, y z^{3}, y^{2} z^{2}, z^{5}\right\}$ and the marked terms of the 24 border basis polynomials are $x z, x^{2} z, x^{3}, x y z, x y^{2}, x z^{2}, x^{2} y z, x^{3} y, x y^{3}, x^{2} y^{2}, x y^{2} z, x y z^{2}, y^{4}, y^{3} z, x z^{3}, x y z^{3}$,

$x y^{2} z^{2}, y^{3} z^{2}, y^{2} z^{3}, y z^{4}, x z^{4}, x z^{5}, y z^{5}$, and $z^{6}$. Since $\mathcal{O}$ contains $x^{2}, y^{2}$ and $z^{2}$ and $f_{2}=y^{2}+y z+z^{2}+x z+x^{2} \in I$ the intersection $\operatorname{LT}_{\sigma}(I) \cap \mathcal{O}$ can never be empty for any term ordering $\sigma$ on $\mathbb{T}^{3}$. Thus the computed border basis cannot arise from a term ordering on $\mathbb{T}^{3}$. 
Remark 3.13. An implementation of the Border Basis Algorithm with Term Marking Strategy is available under the (function) name BB.BBasisForMP in the computer algebra system ApCoCoA (cf. [1, 3]).

Remark 3.14. Let $\sigma$ be a degree compatible term ordering on $\mathbb{T}^{n}$. As noted before in example $3.2 \mathrm{LT}_{\sigma}$ is a marking for any $\mathcal{F} \subseteq P \backslash\{0\}$. The input set $\mathcal{F}$ of the Border Basis Algorithm with Term Marking Strategy can thus be considered being marked by $\mathrm{LT}_{\sigma}$. Now assume that each time the Marked Interreduction is applied to a set $\left\{f_{1}, \ldots, f_{s}\right\} \subset P \backslash\{0\}$ during the execution of the Border Basis Algorithm with Term Marking Strategy the set $\bigcup_{i=1}^{s} \operatorname{Supp}\left(f_{i}\right)=\left\{t_{1}, \ldots, t_{l}\right\}$ is enumerated in such a way that $t_{1}>_{\sigma} \ldots>_{\sigma} t_{l}$ holds. Then the resulting output set of polynomials $\mathcal{F}^{\prime}$ will be marked accordingly to $\mathrm{LT}_{\sigma}$, i.e. each marked term of a polynomial of $\mathcal{F}^{\prime}$ will be the leading term of this polynomial with respect to $\sigma$. This means that in this case the output of the Border Basis Algorithm with Term Marking Strategy will be the same as the output of the Improved Border Basis Algorithm [6, Proposition 21], namely the $\mathcal{O}_{\sigma}\{I\}$-border basis of $\langle\mathcal{F}\rangle_{P}$.

Remark 3.15. The algorithm can indeed encounter the situation that in step (T7) the set $\mathcal{O}$ is not an order ideal. In the following we briefly discuss to reasons why the algorithm cannot produce a border basis for every given input. For this, we assume that the algorithm reaches step $(\mathrm{T} 7)$ and let $\tilde{\mathcal{O}}=\mathcal{U} \backslash\left\{t \cdot \mathrm{MT}_{\mathcal{V}}(v) \mid v \in \mathcal{V}, t \in \mathbb{T}^{n}\right\}$ and $\mathcal{O}=\mathcal{U} \backslash\left\{\operatorname{MT}_{\mathcal{V}}(v) \mid v \in \mathcal{V}\right\}$. Note that $\tilde{\mathcal{O}}$ always forms an order ideal whereas $\mathcal{O}$ is an order ideal if and only if $\mathcal{O}=\tilde{\mathcal{O}}$.

a) One reason for the algorithm to terminate in step (T7) is that the order ideal $\tilde{\mathcal{O}}$ is too small to satisfy $|\tilde{\mathcal{O}}|=\operatorname{dim}_{K}(P / I)$. In this case $\mathcal{O} \backslash \tilde{\mathcal{O}}$ will contain at least one term that does not appear as a marked term of any polynomial in $\mathcal{V}$ and $\mathcal{O}$ will not form an order ideal. An example for this case is given in 3.16 below.

b) Another reason why the algorithm terminates in step (T7) is the scenario when $\tilde{O}$ outlines an order ideal for which no border basis of $I$ exists.

Example 3.16. Let $P=\mathbb{Q}[x, y]$ and $\mathcal{F}=\left\{f_{1}, f_{2}, f_{3}\right\}=\left\{x^{3}, y^{3}, x^{2}+x y+y^{2}\right\}$. The ideal $I=\langle\mathcal{F}\rangle$ is zero-dimensional and $\operatorname{dim}_{\mathbb{Q}}(P / I)=6$. We apply the Border Basis Algorithm with Term Marking Strategy to $\mathcal{F}$ and choose to mark the terms $x^{3}$ in $f_{1}, y^{3}$ in $f_{2}$, and $x y$ in $f_{3}$ in step (T2). Then, already at the beginning of step (T3), the set $\tilde{\mathcal{O}}=\mathcal{U} \backslash\left\{t \cdot \mathrm{MT}_{\mathcal{V}}(v) \mid v \in \mathcal{V}, t \in \mathbb{T}^{n}\right\}=\left\{1, x, y, x^{2}, y^{2}\right\}$ constitutes an order ideal which is too small to support a border basis of $I$. As none of the marked terms of $\mathcal{V}$ will vanish during the following computations the set $\tilde{\mathcal{O}}$ may only shrink further (which it does not do in this example) but cannot become larger. Eventually the algorithm will produce the set $\mathcal{O}=\left\{1, x, y, x^{2}, y^{2}, x y^{2}\right\}$ in step (T7) which is apparently not an order ideal. Here $\mathcal{O} \backslash \tilde{\mathcal{O}}=\left\{x y^{2}\right\}$ reveals which term of $\mathcal{O}$ is a multiple of a marked term of a polynomial in $\mathcal{V}$.

Remark 3.17. The algorithm can be equipped with a backtracking strategy to exhaustively check if a given marking of the input polynomials allows the computation of a border basis. For this to work, the additional book keeping functionality described in remark 3.6 must be included in the Marked Interreduction. If it is then detected in step (T7) that the set $\mathcal{O}$ is not an order ideal, the algorithm can successively go backwards through the log entries of the Marked Interreduction computations, choose different pivot indices $p_{k}$ in step (6), and redo the computation from those points on. If the log entries of the Marked Interreduction are 
exhausted it is then clear that the given marking of the input polynomials does not allow the computation of a border basis.

Remark 3.18. The outcome of the application of the Border Basis Algorithm with Term Marking Strategy highly depends on the input polynomials and the given marking of them. It is clear that if the support of the given input polynomials does not allow to create a marking of the polynomials where the marked terms are terms that can never be a leading term with respect to any term ordering then it is less likely that the computation will yield a border basis that does not arise from a term ordering. On the other hand, in this situation it is also less likely that the algorithm stops in step (T7), especially if the backtracking described in remark 3.17 is used. From an experimental point of view it is thus more fruitful to apply the algorithm to input polynomials that can be marked in many different ways.

\section{Relation to other Border Basis Algorithms}

Let $I \subset P$ be a zero-dimensional ideal. In [10, Mourrain and Trébuchet introduced a very general algorithm which allows the computation of a quotient basis $\mathcal{B}$ of the $K$-vector space $P / I$. In addition, a set of reducing rules which allow projection onto $\langle\mathcal{B}\rangle_{K}$ along $I$ is produced. In this algorithm a choice function refining a reducing graduation $\gamma$ (cf. [10, Definition 2.7]) is used to determine the resulting basis $\mathcal{B}$. For certain choices of $\gamma$ the output $\mathcal{B}$ of this algorithm is an order ideal and the set of reducing rules is a $\mathcal{B}$-border basis of $I$. We observe the following fundamental difference between the algorithm of Mourrain and Trébuchet and algorithm 3.8. The set $\mathcal{B}$ in the algorithm of Mourrain and Trébuchet may grow and shrink as needed during the computation. In contrast to this the set $\mathcal{O}$ in algorithm 3.8 may only shrink in each iteration of step (T7). The latter behaviour ensures that no marked term of any marked polynomial in the initial input set $\mathcal{F}$ can appear in the order ideal $\mathcal{O}$.

Let $I$ be generated by a finite set of polynomials $\mathcal{F} \subset P$. Braun and Pokutta presented another more general algorithm to compute an $\mathcal{O}$-border basis of $I$ for an order ideal $\mathcal{O}$ in 2 . In contrast to algorithm 3.8 not only one specific $\mathcal{O}$-border basis is computed but instead an $L$-stable span of $\langle\mathcal{F}\rangle_{K}$ (cf. [6, Definition 10]) where $L=\left\langle\mathbb{T}_{\leq d}^{n}\right\rangle_{K}=\left\langle\left\{t \in \mathbb{T}^{n} \mid \operatorname{deg}(t) \leq d\right\}\right\rangle_{K}$ for some $d \in \mathbb{N}$ is produced which contains all possible $\mathcal{O}$-border bases of $I$. After choosing an admissible order ideal $\mathcal{O}$ the polynomials of the $\mathcal{O}$-border basis are then selected from this $K$-vector space in the last step of the algorithm by a basis transformation. Due to the dependency on the shape and the marking of the input polynomials, the application of the Border Basis Algorithm with Term Marking Strategy always results in a very specific computation which means that it does not necessarily produce such a $K$-vector space during its execution. This behavior can result in a faster running time at the expense of generality as shown in the following example.

Example 4.1. Let $K=\mathbb{Q}, P=\mathbb{Q}[x, y], \mathcal{F}=\left\{x^{2}-y, x^{2} y+y^{3}-x-y\right\}$, and $I=\langle\mathcal{F}\rangle$. Let $\sigma$ denote the standard Lex term ordering on $\mathbb{T}^{2}$ where $x>_{\sigma} y$ and let $\tau$ denote the Lex term ordering on $\mathbb{T}^{2}$ where $x<_{\tau} y$. Since the reduced $\sigma$ - and $\tau$-Gröbner bases of $I$ are $\left\{x-y^{3}-y^{2}+y, y^{6}+2 y^{5}-y^{4}-2 y^{3}+y^{2}-y\right\}$ and $\left\{y-x^{2}, x^{6}+x^{4}-x^{2}-x\right\}$, respectively, algorithm 4.3 of $\left[2\right.$ must produce the computing universe $L=\mathbb{T}_{<6}^{2}$ for a stable span computation during its execution. Because of the shape of the 
polynomials of $\mathcal{F}$ the Border Basis Algorithm with Term Marking Strategy cannot be used to compute neither the $\mathcal{O}_{\sigma}\{I\}$-border basis nor the $\mathcal{O}_{\tau}\{I\}$-border basis of $I$. It can be shown that regardless of the chosen marking of $\mathcal{F}$ and the chosen enumeration of terms for the application of the Marked Interreduction the result of the computation in this case will always be the same $\mathcal{O}$-border basis of $I$. But in contrast to the computing universe $L=\mathbb{T}_{\leq 6}^{2}$ as in the case of algorithm 4.3 of [2] the final computing universe during the execution of the Border Basis Algorithm with Term Marking Strategy is the order ideal $\mathcal{U} \subset \mathbb{T}_{\leq 4}^{2}$ spanned by $y^{4}, x y^{3}, x^{2} y^{2}$, and $x^{3} y$.

\section{REFERENCES}

[1] The ApCoCoA Team, ApCoCoA: Approximate Computations in Commutative Algebra, available at http://www. apcocoa.org

[2] G. Braun and S. Pokutta, Border bases and order ideals: a polyhedral characterization, arXiv: math $\backslash 0912.1502 \mathrm{v} 2$.

[3] The CoCoA Team, CoCoA: a system for doing Computations in Commutative Algebra, available at http://cocoa.dima.unige.it

[4] D. Heldt, M. Kreuzer, S. Pokutta, and H. Poulisse, Approximate computation of zerodimensional ideals, J. Symb. Comput. 44 (2009), 1566-1591.

[5] A. Kehrein and M. Kreuzer, Characterizations of border bases, J. Pure Appl. Alg. 196 (2005), $251-270$

[6] A. Kehrein and M. Kreuzer, Computing border bases, J. Pure Appl. Alg. 205 (2006), 279-295.

[7] A. Kehrein, M. Kreuzer and L. Robbiano, An algebraist's view on border bases, in: A. Dickenstein and I. Emiris (eds.), Solving Polynomial Equations: Foundations, Algorithms, and Applications, Springer, Heidelberg 2005, 169-202.

[8] M. Kreuzer and L. Robbiano, Computational Commutative Algebra 2, Springer, Heidelberg 2005.

[9] B. Mourrain, A new criterion for normal form algorithms, AAECC Lecture Notes in Computer Science 1719 (1999), 430-443.

[10] B. Mourrain and P. Trébuchet, Generalised normal forms and polynomial system solving, Proceedings of the 2005 international symposium on Symbolic and algebraic computation affiliation (2005), 253-260.

[11] H.J. Stetter, Numerical Polynomial Algebra, SIAM, Philadephia 2004.

Fakultät für Informatik und Mathematik, Universität Passau, D-94030 Passau, GerMANY

E-mail address: Stefan.Kaspar@uni-passau.de 